\title{
Power Optimized Quantum Data Aggregation Scheduling
}

\author{
S. Madhavi* \\ PVP Siddhartha Institute of Technology, India \\ *mmadhavi@pvpsiddhartha.ac.in
}

\begin{abstract}
Communication Networks have limited resources like bandwidth and battery power. Hence efficient node scheduling protocols and communication protocols are needed to maximize the network throughput. In this paper, we discussed an optimal data aggregation scheduling problem for quantum networks. To optimize the utilization of the network bandwidth much research is done to find schedules with minimum frame length. In this paper we proposed a method for finding efficient quantum node scheduling. The schedule contains a set of slots where each slot is allotted to one or more quantum nodes for transmitting at the same time. Adjacent Quantum nodes can create entanglement for transmitting the data. The quantum nodes entangles with its neighbor quantum nodes during the slot allotted to them. Loss and distortion are the two major problems in free space channels transmission. The optical noise produced when two or more nodes are transmitting data concurrently interferes with the actual data. Hence the optical signal to noise ratio OSNR ie Square root of the signal power divided the square root of the noise power is used as a threshold on concurrent transmitters. In this proposed method we used OSNR for finding a set of nodes that can entangle concurrently for aggregating the data to the base station. Entanglement rate of each node is used as a metric for finding a schedule that maximizes the quantum network throughput. We derive a Power Optimized Maximal Link Entanglement Rate based Scheduling Method which maximizes the quantum network throughput and also increases the network life time.
\end{abstract}

Keywords: EPR, Quantum Teleportation, Quantum Network, Quantum Entanglement, OSNR

\section{Introduction}

Communication plays an important role in day to day activities. Nodes acquire data and aggregates data to the base station securely and accurately. Since battery power and bandwidth are the two important resources to be optimized efficient data transmission, power optimization [30-32] and node scheduling methods play an important role in data communication. Much research is done in finding such efficient scheduling protocols to maximize the network throughput [28]. Due to advancement in the field of communications many novel protocols in the field of quantum computation [1] and quantum networks [2,3] are implemented. Various applications in quantum networks [11] are multiparty shared secrets which relies on the laws of physics [12,13], distributed quantum computing [14], improved sensing [15, 16], long-distance efficient entanglement distribution $[4,5]$ using quantum repeaters $[6,7]$. Currently Long distance communication is one of the major research topic of interest. H. V. Nguyen proposed a novel method for the quantum Internet. He proposed a generalized quantum network coding for large-scale quantum communication networks. Quantum Teleportation is another research field where many security protocols are studied.

Received (January 15, 2018), Review Result (March 25, 2018), Accepted (April 1, 2018)

${ }^{*}$ Corresponding Author 
Q.-C. Sun proposed method for quantum teleportation with independent sources where there is a prior entanglement distribution over the network. Recently with the advancements in quantum technologies J. Yin discovered long distance quantum communication. His method is a satellite-based entanglement distribution over 1200 kilometers which is a major achievement in the field of quantum communications. In June 2017, Chinese physicists led by Pan Jianwei measured entangled photons over a distance of $1203 \mathrm{~km}$ between two ground stations. T. Bacinoglu, B. Gulbahar, and O. B. Akan proposed a method for constant fidelity entanglement in quantum communication networks. Since data loss is natural in network communications, repeaters play a major role for successful data communications. R. Van Meter studied on the path selection for quantum repeater networks.

Changai Chen and Yanwen $\mathrm{Xu}$ proposed an improved Quantum Ant Colony Optimization Algorithm for Solving Complex Function Problems. Much research is also done in the field of clustering. Amita Yadav, Suresh Kumar proposed an optimization Algorithm for Cluster Head Selection in Wireless Sensor Networks. A node in the network forms EPR pair with its 1-hop neighbor over a quantum communication channel. Quantum entanglement swapping makes two independent quantum entangled states [24] become entangled without direct interaction [18-26]. Quantum teleportation of entangled states [24] was demonstrated in discrete- variable optical systems [18, 19], and extended to continuous-variable systems [22-26].

Initially quantum cryptography was studied by Bennett-Brassard 1984 (BB84) where the cryptography key is distributed between two distant nodes with the transmission of a quantum state sequence. Quantum teleportation enables transportation of an unknown quantum state to a remote station by using an entangled state as the quantum resource [1826]. Hence using quantum entanglement, teleportation, and swapping, a quantum communication network is formed where the information is transmitted by quantum states. Little research is done in the field of quantum network node scheduling for transmitting so as to maximize the throughput and minimize the power and bandwidth utilization.

Hence in this paper we proposed a Power Optimized Maximal Link Entanglement Rate based scheduling Method for quantum networks. When a signal (data and noise) traverse a certain distance it becomes weak. Optical amplifiers are used to strengthen the signal, which in turn strengthens the noise too and finally the receivers cannot distinguish the signal from the noise. Unlike traditional scheduling methods which uses Dijsktra's algorithm, our proposed method uses entanglement generation probability, rate and OSNR as metrics to find which next node to transmit so as to increase the throughput of the network. The proposed method uses optimal power and bandwidth.

In Section 2.0 we introduced the proposed network model for computing a schedule to maximize the throughput in quantum entangled networks. In Section 3.0 the scheduling method is discussed. In Section 4.0 we discussed the implementation and conclusion. In Section 5.0 we proposed the future work.

\section{Quantum Network Model}

Let us consider a Quantum network denoted by a graph $\mathrm{G}(\mathrm{V}, \mathrm{E})$ with $\mathrm{V}=\{\mathrm{Vi}\} \mathrm{i}=1$..N where $\mathrm{Vi}, \mathrm{Vj} € \mathrm{~V}$ and denotes the set of mobile quantum nodes, $\mathrm{N}$ is the total number of nodes. The nodes are power adaptable. In the proposed quantum network there are two types of links are used between the adjacent nodes for communication.

They are

-Quantum links which are represented by thick lines to transfer quantum state and

-Wireless link which are represented by thin lines to transfer the classical data. 
A simple quantum network model is shown in Figure 1.0.

For the implementation, we assumed a complex network with varying number of nodes and varying degree at each node. At present quantum communication can be used to communicate with the satellites. Establishing the connection for long distances is difficult which can be overcome by the usage of the repeater. For example, for every quantum distance of 1,000 km long optical telecommunication there will be approximately a loss of $0.2 \mathrm{~dB} / \mathrm{km}$. Secured transmission is the main aim of quantum networks. Such secured transmission in a large quantum network can be possible by aggregating the data from the interconnecting neighboring nodes within the short-distance.

In the proposed method we adopted the idea of using quantum entanglement as a communication resource between two users who are separated by a short distance. We adopted heralded quantum entanglement links between several neighbor nodes within short distance for generating quantum entanglement in the quantum communication network. Finally, all the intermediate quantum nodes aggregate data to the base station.

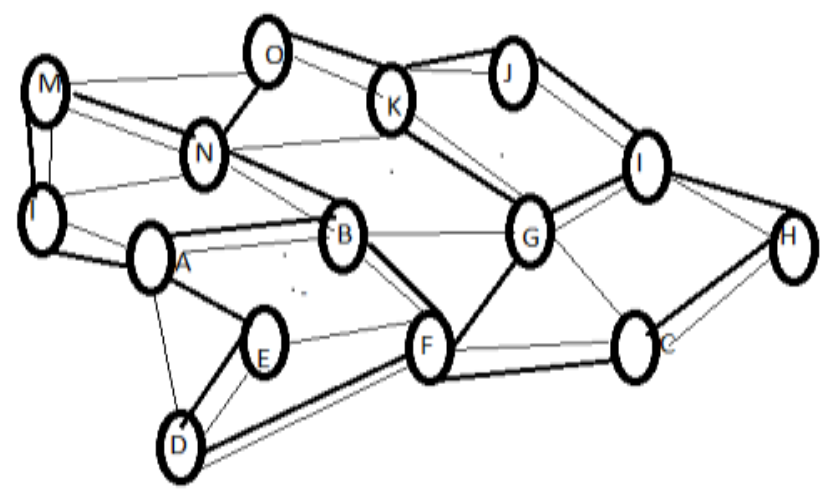

Figure 1. Quantum Network with Wireless and Quantum Links

An edge eij between node $\mathrm{i}$ and node $\mathrm{j}$ exist iff a quantum and wireless links simultaneously exist between them. Hence in Figure 1.0 node A and B can transfer information by quantum states since there is a 1-hop-by-1-hop quantum channel and a wireless channel between them. Similarly, there is no quantum channel between nodes A and node $\mathrm{D}$, hence they are not adjacent nodes.

Nodes $\mathrm{A}$ and $\mathrm{C}$ are called as concurrent quantum transmitters since we considered a distance of 3-hop as interference free distance between any concurrent transmitters. For any arbitrary couple of nodes $\mathrm{Vi}, \mathrm{Vj}$, if it exists ei, $\mathrm{j}$ belongs to $\mathrm{E}$ then $\mathrm{Vi}$ and $\mathrm{Vj}$ are defined as adjacent nodes. The value of the edge between two nodes denotes the number of shared EPR pairs. A quantum link exists between two nodes as long as there are shared EPR pairs with them otherwise the link will be terminated. Let the Graph $\mathrm{G}$ contains of $\mathrm{K}$ source-destination (Alice-Bob) pairs $(\mathrm{Aj}, \mathrm{Bj}), 1 \leq \mathrm{j} \leq \mathrm{K}$, in $\mathrm{V}$.

Dij denote the length of the optical link and $\mathrm{Ti}, \mathrm{j}$ denotes the average entanglement time between node vi and vj respectively. The nodes transmit information by quantum states. The topology of the network changes dynamically whenever

- A node adds or leaves the network or

-When the EPR pairs owned by two nodes are exhausted.

If a node has some data to aggregate to the base station it is called "active node". Every node shares EPR pairs with its neighbor within its communication range (called 1-hop distance i.e., 50cms). Quantum teleportation is used to transfer a quantum state from one node to another via entangled particles, such as EPR pairs [11]. Figure 2.0 [20] shows the optical network with heralding cavity and entangling cavity. 
Heralding cavity detects the entanglement generation and entangling cavity makes photon to the mode of a single-mode optical fiber [20]. Let three nodes Alice, Bob and Charlie share a Heralded entanglement separated by small distance. If Alice wants to transmit to Charlie then first Alice create entanglement with Bob and then Bob create entanglement with Charlie. Hence indirectly Alice creates entanglement with Charlie. Similarly, every node in the network aggregates data to the nearest optimal 1-hop neighbor (denoted as a head-node). For data aggregation in this paper we proposed a novel solution for improving the performance of quantum network.

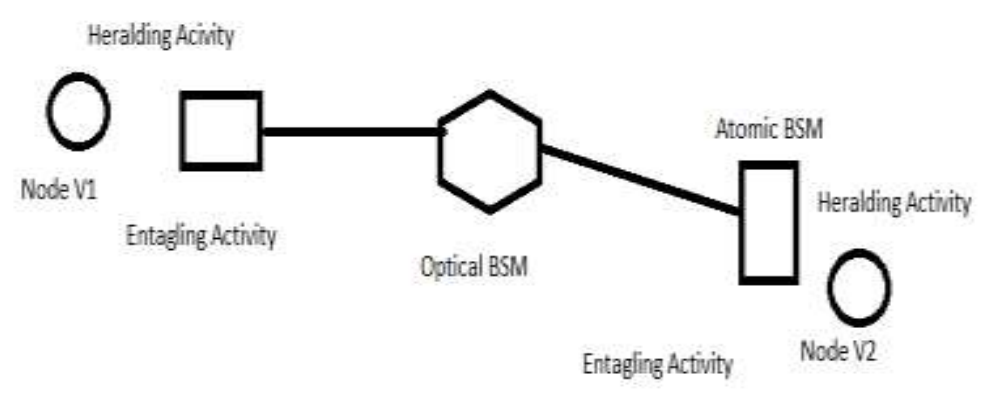

Figure 2. Optical Network with Heralding Entanglement

The proposed data aggregation scheduling method consists of the following steps:

-Identification of optimal head node

-Entanglement formation.

- Constructing a schedule.

-Finding an optimal schedule.

An entanglement is formed only when

-The probability for creating a successful link is above a threshold value.

-The OSNR perceived at the head-node is above a threshold value.

-Minimum EPR pairs are consumed for transferring quantum states.

Once the entanglement is formed between two nodes say Alice and Bob then one particle of the Alice has been entangled with the Bob. One or more nodes can establish entanglement concurrently. In order to reduce the interferences at the receiver, every two nodes with a minimum distance of 3-hop apart are selected as concurrent transmitters. A set of schedules are prepared where each schedule consists of one or more number of slots. In each slot one or more non conflicting nodes can transmit concurrently. After determining the concurrent nodes for transmitting in a time slot, the selected nodes are labeled as "Visited ". In order to maintain the principle of fairness a visited node do not participate in the race for obtaining a time slot until all the nodes are scheduled at least once. Since nodes are mobile the total number of nodes in the network changes dynamically. From a set of constructed schedules, a schedule which yields is called as the optimal schedule.

-Maximum Network life time,

-Highest Entanglement rate

-Optimum Power and width utilization 
The label of a node remains unvisited if it is not allotted with any slot in the schedule. The procedure is repeated until all the nodes aggregate data to the base station. In this paper we proposed a novel Power Optimized Maximal Link Entanglement Rate based Scheduling Method for the quantum network which is based on the entanglement rate at a node. The nodes are power adaptable and for efficient utilization of the available bandwidth, two or more nodes which are geographically separated by a predetermined distance and with optimal optical signal-to-noise ratio OSNR are allowed to transmit concurrently in one time slot. A schedule which makes maximum number of transmissions in one cycle is called optimum schedule.

An optimal schedule always maximizes the throughput and minimizes the utilization of the power and bandwidth. The link entanglement generation probability denotes the probability for successfully generating an entanglement between two adjacent nodes vi and vj through optical link [19]. The link entanglement generation time denotes the average time required for successfully generating an entanglement between two adjacent nodes vi and vj through optical link [19]. The link entanglement rate denotes the average number of successful entanglement generations within the unit time between two adjacent nodes vi and vj through optical link which can be successfully used for teleportation given the quantum memory coherence time [19].

$$
O S N R=\frac{p_{i}}{\eta_{i}^{0}+\sum_{j \in N} \Gamma_{i j} p_{j}}
$$

Where $\mathrm{Pi}$ and $\mathrm{Pj}$ are the transmitter and receiver powers, $\mathrm{N}$ denotes the available channels for transmission $\Gamma_{i j}$ represents the number of transmitters permitted to transmit concurrently during the same slot when pi is transmitting to $\mathrm{pj} . \eta_{i}^{0}$ represents the noise due to various effects. ONSR decides the number of concurrent transmitters. When a set of nodes are allowed to entangle concurrently, they produce noise and this noise may effect the quality of the data transmitted. For a successful entanglement the OSNR value perceived should be limited to a threshold value. Hence OSNR acts as the threshold upon the maximum number of nodes which are allowed to entangle concurrently with the neighbor quantum nodes in the same time slot. $\beta$. Minimum frame lengths maximize the network throughput. As the nodes are power adaptable, we used power optimization techniques to allow one or more nodes to entangle concurrently in such a way that the total perceived OSNR I below a threshold value.

\section{Optimal Data Aggregation using Quantum Entanglement}

Today Quantum internets had become the most interesting topic for many researchers. Untill bb84 protocol people used lengthy key cryptographic methods for secured transmission. With the rapid changes in the quantum technologies, new techniques like quantum teleportation, entanglement had evolved where information can be transmitted to other node in a network without the actual physical transmission. The quantum nodes can share quantum information in a quantum network using entangled links between them. These links can be of shorter distance like a few meters and lengthy like 1200 kilometers. All the entangled shared qubits form a quantum communication channel in a quantum network. Generally, Sensors have a limited memory, processing capability, battery power and bandwidth. In this paper for a distributed warless quantum sensor network we would like to propose a data aggregation protocol for effective utilization of these limited resources. The sensors use the local state information at the nearest 1-hop neighbor to find the best parameters for increasing the entanglement rate at each node. 
To share information between two nodes like Alice and Bob which are separated by a large distance like a few tens of kilometers repeaters are used to create entanglement between their qubits.

In this section we describe the proposed Maximal Link Entanglement rate based data aggregation scheduling method for Quantum Network. The main objective is to maximize the network throughput and minimize the frame length. The proposed method iterates until there are no new schedules found. Each iteration continues until all the nodes are visited at least once.

Let there are $\mathrm{N}$ nodes in the quantum network

Let there are 1..m 1-hop neighbors for each node

Let each ith node possess $E_{\text {num }}^{\mathrm{i}}$ EPR pairs to share with its 1-hop neighbors while establishing the entanglement.

A. Repeat until no new schedule is found

i. Repeat until all the nodes are scheduled at least once

I. For each $\mathrm{i}=1$ to Max-hop do

II. Select a node $\mathrm{a}_{\mathrm{i}}$ and add it to the slot in the current schedule

III. Increment schedule sh by 1

IV. For $\mathrm{k}$ in [ $\mathrm{a}_{\mathrm{i}}$ and 3-hopth neighbors of $\mathrm{a}_{\mathrm{i}}$ which are not visited yet] and $\mathrm{k}$ not interfering with any visited nodes do

V. For $\mathrm{j}=1$ to Max_Deg(k) do

VI. Let $b_{j}$ denote the jth 1-hop neighbor of $k$ which is not visited yet

VII. If quantum and wireless links exists between nodes $\mathrm{k}$ and $\mathrm{bj}$ then

a. Find the link entanglement generation probability[19] $\mathrm{p}$ at $\mathrm{k}$ using

$$
p=\frac{1}{2} v_{0}\left(p e^{-d /\left(2 L_{0}\right)}\right)^{2}
$$

Where V0 denotes the optical BSM efficiency at each node and $\mathrm{d}_{\mathrm{ij}}$ denotes the length link and L0 denotes the attenuation length of the optical fiber

b. If $\mathrm{p}$ is $>=6 \%$ then

Calculate OSNR at node $\mathrm{k}$

$$
O S N R=\frac{p_{i}}{\eta_{i}^{0}+\sum_{j \in N} \Gamma_{i j} p_{j}}
$$

Where $\mathrm{p}_{\mathrm{i}}$ and $\mathrm{p}_{\mathrm{j}}$ refers to powers at transmitter and receiver

$$
\text { If OSNR > 20dB then }
$$

$$
T=\frac{\overline{p_{i j}} T_{i j}^{f}+p_{i j} T_{i j}^{s}}{p_{i j}}
$$

Where $T_{i j}{ }^{f}$ denotes the failure and $T_{i j}{ }^{s}$ denotes the success rate of creating an entanglement between nodes say $i$ and $j$.

Let $\mathrm{T}_{\mathrm{ch}}$ denotes the quantum memory coherence time.

Link entanglement rate is less than 0 if $\mathrm{T}_{\mathrm{ch}}$ is less than the average time elapsed between the atom photon generation and the ack reception otherwise it is equal to $1 /$ Tij.

Let $\gamma$ denotes the link entanglement rate threshold.

If link entanglement rate $>\gamma$ then

Establish an entanglement between $\mathrm{k}$ and $\mathrm{b}_{\mathrm{j}}$

Let EL(k,bj) represent the established Entangled Link

Decrement the $E^{\mathrm{k}}$ num by 1 .

Append bj to the schedule [sh][k][slot] 
Else

Increment concurrenttransmtters $\Gamma$ kbj by 1

Update the entanglement rate for node $\mathrm{k}$

end if

Since the entanglement probability failed

Increase power pi by a small factor $\delta$ at bj

I. End For j

Repeat VIII

II. End For $\mathrm{k}$

III. Increment slot by 1

IV. End for i

V. End Repeat

B. End Repeat

C. Throughput achieved $=$ total number of transmissions in all slots in one schedule / frame length for a given number of nodes $\mathrm{N}$.

D. After finding all the schedules the optimal schedule has to be identified. Since the efficient schedule contains maximum number of transmissions and minimum frame lengths we use

Sort the schedules in the descending order of the function of minimum slots, maximum concurrent transmitters, maximum entanglement rate

E. Select the first schedule which contains the transmission schedule which is called as the optimal schedule

F. End

The above method is used to find the optimal schedule for the quantum networks.

\section{Conclusion}

In this paper, we designed an optimal data scheduling method to aggregate data to the base station in quantum networks.

The proposed method finds a schedule which

a. Optimizes the bandwidth utilization

b. Increases the network life time

c. Optimal sharing of the EPR pairs

d. Minimum frame length with maximum entanglement rate at the selected nodes

e. Since the nodes are power adaptable minimum power requirements at each node and hence the nodes lives longer.

Each mobile station can tune to its required transmission power. The average time slots increase linearly as the number of nodes increases. We assumed a network of $250 \mathrm{mx} 250 \mathrm{~m}$ where the nodes are uniformly distributed in a random way. We tested the protocol in a quantum network consisting of 50,100, 150, 200, and 250 numbers of nodes with different topologies and varying degree 2, 4, 6. EPR pairs are shared between the nodes which are within their transmission range. Initially a value between 500 to 1000 number of EPR pairs is assumed between the nodes. A bi-directional wireless channel is assumed between the node and each node has a bandwidth of $11 \mathrm{Mbps}$. In our experiment, the average pump power for each source was about $80 \mathrm{~mW}$, leading to a probability of creating an entangled pair per laser pulse of around 6\%. The proposed method optimizes the perceived OSNR so as to reduce the interferences at the receiver produced by the concurrent transmitters. The proposed method finds an efficient schedule for the nodes to transmit using entanglement swapping between pair of nodes so as to maximize the quantum network throughput. The nodes which have the highest probability for creating 
the entanglement and which can produce highest entanglement rate are chosen so as to increase the network throughput. The proposed scheduling method is different from the traditional methods which uses the distance as a metric to find the efficient path. Hence the proposed method can be used in data communications as a node scheduling method.

\section{Future Work}

Since the long distance coverage is more essential in today's applications the proposed method can be extended to long distance quantum communication and distributed quantum computation. We wanted to study a protocol for establishing secure quantum communication between distant locations which requires distributed entangled states between nodes like in DLCZ protocol [34] for a multi-node setting between multiple nodes over long distances. In future we wanted to propose methods for efficient communication in a quantum internet using quantum repeaters for avoiding the loss of data.

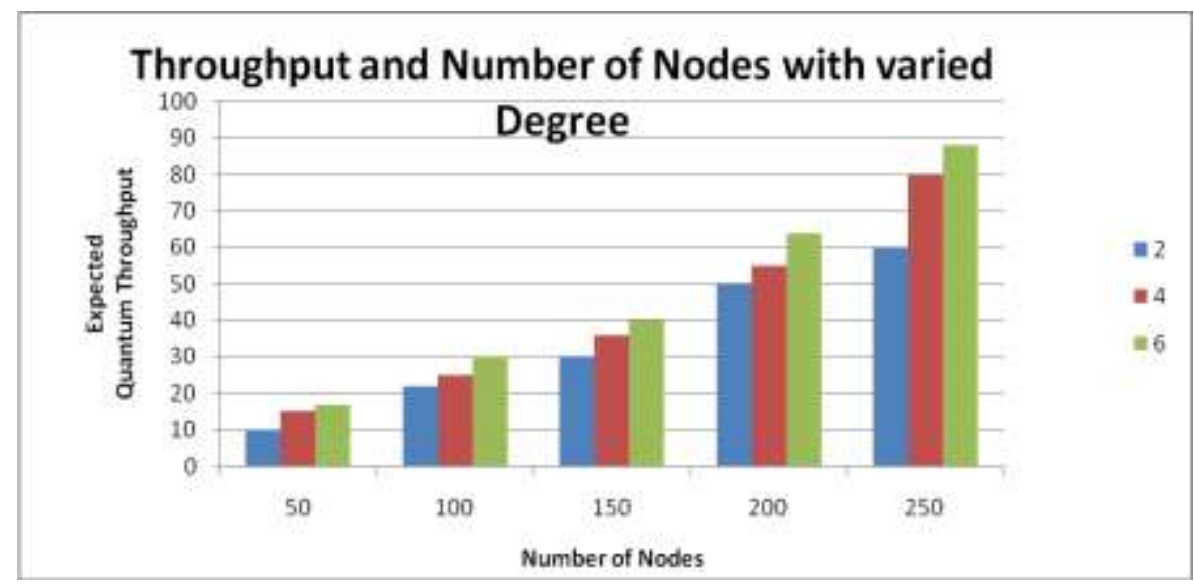

Figure 3. Expected Quantum Throughput Vs Number of Nodes

\section{Acknowledgment}

Project supported by the SERO UGC of India (MRP-6520/16).

\section{References}

[1] A. Hellemans, "Europe bets AC1 billion on quantum tech [News]", IEEE Spectr., vol. 53, no. 7, (2016) July, pp. 12-13.

[2] H. J. Kimble, 'The quantum internet”, Nature, vol. 453, no. 7198, June 8, pp. 1023-1030.

[3] H. V. Nguyen, "Towards the quantum Internet: Generalized quantum network coding for large-scale quantum communication networks",' IEEE Access, vol. 5, (2017), pp. 17288-17308.

[4] Q.-C. Sun, "Quantum teleportation with independent sources and prior entanglement distribution over a network", Nature Photon., vol. 10, no. 10, (2016) October, pp. 671-675. Quantum Inf. Process. 12, 331$344(2013)$

[5] J. Yin, "Satellite-based entanglement distribution over 1200 kilometers", Science, vol. 356, no. 6343, pp. 1140-1144.

[6] H.-J. Briegel, W. Dür, J. I. Cirac and P. Zoller, "Quantum repeaters: The role of imperfect local operations in quantum communication”, Phys. Rev. Lett., vol. 81, (1998) December, pp. 5932-5935.

[7] W. Dür, H.-J. Briegel, J. I. Cirac and P. Zoller, "Quantum repeaters based on entanglement purification", Phys. Rev. A, Gen. Phys., vol. 59, (1999) January, pp. 169-181.

[8] T. Bacinoglu, B. Gulbahar and O. B. Akan, "Constant fidelity entanglement in quantum communication networks", Proc. IEEE Global Telecommunication. Conf. GLOBECOM, (2010) December, pp. 1-5.

[9] R. Van Meter, "Path selection for quantum repeater networks", Netw. Sci., vol. 3, no. 1, (2013) December, pp. 82-95.

[10] C. H. Bennett, G. Brassard, C. Crépeau, R. Jozsa, A. Peres and W. K. Wootters, "Teleporting an unknown quantum state via dual classical and Einstein-Podolsky-Rosen channels", Phys. Rev. Lett., vol. 70, (1993) March, pp. 1895-1899. 
[11] H. J. Kimble, "Nature", vol. 453, no. 1023, (2008).

[12] A. K. Ekert, "Physical Review Letters", vol. 67, no. 661, (1991).

[13] C. H. Bennett and G. Brassard, "Theoretical Computer Science", vol. 560, no. 7, (2014).

[14] J. Cirac, A. Ekert, S. Huelga and C. Macchiavello, "Physical Review A", (1999).

[15] S. Madhavi, "Quantum Boson Data Aggregation Scheduling in UOSN", International. Journal of. Control and Automation, vol. 8, no. 4, (2015).

[16] D. Gottesman, T. Jennewein and S. Croke, "Physical Review Letters", vol. 109, 0705032012.

[17] P. Komar, E. M. Kessler, M. Bishof, L. Jiang, A. S. Srensen, J. Yes, and M. D. Lukin, "Nature Physics", vol. 10 , no. 582 , (2014), arXiv:arXiv: $1310.6045 v 1$.

[18] J. Fitzsimons Broadbent and E. Kashe, 2009 50th Annual IEEE Symposium on Foundations of Computer Science IEEE, (2009), pp. 517-526.

[19] X. Su, C. Tian, X. Deng, Q. Li, C. Xie and K. Peng, "Quantum entanglement swapping between two multipartite entangled states", https://arxiv.org/pdf/1612.04479.pdf, (2016).

[20] M. Caleffi, "End-to-End Entanglement Rate: Toward a Quantum Route Metric", 978-1-5386-39207/17/\$31.00, IEEE, (2017).

[21] M. Caleffi, "Optimal Routing for Quantum Networks, In-situ and Remote Sensors toward Integrated Earth Sensing", Journal of Sensors.

[22] F. Sciarrino, E. Lombardi, G. Milani and F. De Martini, Phys. Rev. A 66, 024309, (2002).

[23] H. de Riedmatten, I. Marcikic, J. A. W. van Houwelingen, W. Tittel, H. Zbinden and N. Gisin, Phys. Rev. A71, 050302(R), (2005).

[24] R. E. S. Polkinghorne and T. C. Ralph, Phys. Rev. Lett.83, 2095, (1999)

[25] S. M. Tan, Phys. Rev. A 60, 2752, (1999).

[26] P. van Loock and S. L. Braunstein, Phys. Rev. A 61,010302(R), (1999).

[27] X. Jia, X. Su, Q. Pan, J. Gao, C. Xie and K. Peng, Phys.Rev. Lett. 93, 250503, (2004).

[28] N. Takei, H. Yonezawa, T. Aoki and A. Furusawa, Phys.Rev. Lett. 94, 220502, (2005).

[29] S. Madhavi and T. H. Kim, "A Dynamic and Distributed Scheduling for Data Aggregation in Ubiquitous Sensor Networks Using Power Control", Hindawi Publishing Corporation International Journal of Distributed Sensor Networks, (2013).

[30] C. Chen and Y. Xu, "An Improved Quantum Ant Colony Optimization Algorithm for Solving Complex Function Problems", International Journal of Multimedia and Ubiquitous Engineering, vol. 10, no. 11, (2015), pp. 193-204.

[31] A. Bashardoust, M. Farrokhifar, A. Yousefzadeh Fard, A. Safari and E. Mokhtarpour, "Optimum Network Reconfiguration to Improve Power Quality and Reliability in Distribution System", International Journal of Grid and Distributed Computing, vol. 9, no. 4, (2016), pp. 101-110.

[32] A. Yadav and S. Kumar, "A Teaching Learning Based Optimization Algorithm for Cluster Head Selection in Wireless Sensor Networks", International Journal of Future Generation Communication and Networking, vol. 10, no. 1, (2017), pp. 111-122.

[33] L. M. Duan, M. D. Lukin, J. I. Cirac and P. Zoller, "Long-distance quantum communication with atomic ensembles and linear optics", Nature, vol. 414, (2001), pp. 413-418.

[34] Y. Li, Phd Thesis report "Design of Quantum Repeaters". 
International Journal of Grid and Distributed Computing

Vol. 11, No. 7 (2018) 